\title{
Modelling Hysteresis of Soft Core Materials using Permeance-Capacitance Analogy for Transient Circuit Simulations
}

\author{
M. Luo, D. Dujic, and J. Allmeling
}

This material is posted here with permission of the IEEE. Such permission of the IEEE does not in any way imply IEEE endorsement of any of EPFL's products or services. Internal or personal use of this material is permitted. However, permission to reprint / republish this material for advertising or promotional purposes or for creating new collective works for resale or redistribution must be obtained from the IEEE by writing to pubs-permissions@ieee. org. By choosing to view this document, you agree to all provisions of the copyright laws protecting it. 


\title{
Modelling Hysteresis of Soft Core Materials using Permeance-Capacitance Analogy for Transient Circuit Simulations
}

\author{
Min Luo, Drazen Dujic \\ Power Electronics Laboratory \\ École Polytechnique Fédérale de Lausanne \\ Station 11, CH-1015 Lausanne \\ Email:min.luo@epfl.ch,drazen.dujic@epfl.ch \\ URL: http://pel.epfl.ch
}

\author{
Jost Allmeling \\ Plexim GmbH \\ Technoparkstrasse 1, CH-8005 Zürich \\ Email: allmeling@plexim.com \\ URL: http://www.plexim.com
}

\section{Keywords}

$<<$ hysteresis $>>,<<$ soft material $>>,<<$ magnetic circuit $>>,<<$ permeance-capacitance $>>$.

\begin{abstract}
Magnetic components using soft core materials are widely used in power electronic converters. The hysteresis effect of the material leads to power loss and harmonic distortion. If this effect can be modeled in system-level time-domain simulation, the performance of the magnetic component in combination with power converters can be predicted more accurately during the design phase. This work proposes an approach to model the hysteresis effect of soft magnetic materials in magnetic circuits based on the permeance-capacitance analogy.
\end{abstract}

\section{Introduction}

In power electronic circuits, soft materials like ferrite and nanocrystaline are commonly used for transformer and filter inductor cores. Magnetic hysteresis of the core materials directly leads to power loss and nonlinear behaviour of the magnetic components. If this effect can be modeled into time-domain simulation of power electronic circuits, the performance of the magnetic component with respect to different design configurations can be sufficiently evaluated before constructing a prototype, so that the number of hardware iterations can be, possibley, reduced.

Magnetic circuits based on permeance-capacitance analogy introduced by [1] have been proved to be practical for fast circuit simulation in the work of [2], [3] and [4]. Fig. 1(a) and Fig. 1(b) demonstrate the model of a four-winding transformer and its gyrator-type interface with the electrical circuit. The

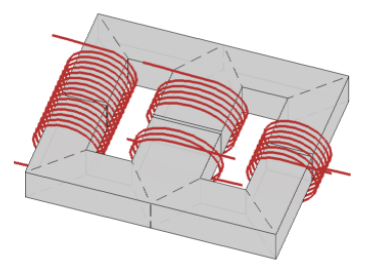

(a)

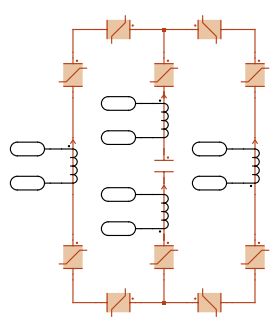

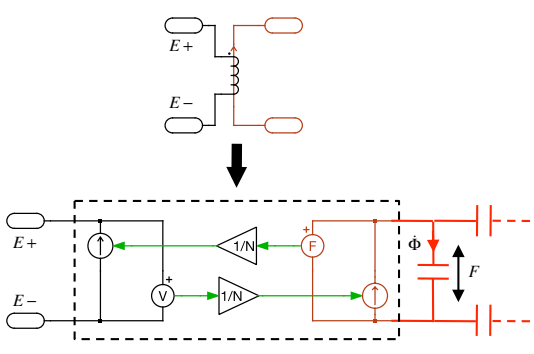

(b)

Fig. 1: (a) Permeance-capacitance magnetic circuit of a four-winding transformer (b) Gyrator interface between magnetic- and electrical circuit 
authors of [2] have also implemented a hysteresis core block based on the classical Preisach model with the formulation proposed by [5]. In this way, one can dynamically simulate the hysteresis effect under arbitrary excitation conditions.

The classical Preisach model only takes into account the irreversible magnetisation, which is sufficient for representing materials like silicon steel with a wide hysteresis loop. The authors of [6] have successfully simulated the inrush current of a three-phase line-frequency transformer with magnetic circuit model. For most soft materials with narrow hysteresis loops, however, the classical Preisach model will produce considerable error [7]. In order to adapt the Preisach model to soft core materials more generally, in [7] it was suggested to add a reversible component, for which a process of parameter identification has been described in the work of [8]. Using this model, the authors of [9] were able to reproduce the hysteresis loop of simple two-winding transformer made by NiFe material in an electrical equivalent circuit model. For modelling complex magnetic structures however, no publication has introduced this approach to magnetic circuits so far. As an extension to the work done by [2], this paper proposes an approach to implement the combined hysteresis model as a permeance-capacitance based magnetic circuit, which is able to simulate hysteresis effects of soft core materials accurately. Also, a parameter identification scheme is introduced whcih is simpler than the one presented in [8].

\section{Classical Model}

In permeance-capacitance based magnetic circuits, magnetic fluxes are assumed to be confined in predefined tubes, represented by the network of core blocks (permeance) in order to approach the real geometry of the target device. The across-variable of magnetic circuit is magneto-motive force $F(\mathrm{MMF})$ while the flow-variable is magnetic flux's rate of change $\dot{\Phi}=d \Phi / d t$. The core blocks in the magnetic circuit behaves like a magnetic "capacitor" with permeance value $P$. Its magnetic "voltage" $F$ comes from the integration of the magnetic "current" $\dot{\Phi}$, as shown in Fig. 1(b). The relation between $F$ and $\dot{\Phi}$ is given as:

$$
F=\frac{1}{P} \int \dot{\Phi} \cdot d t
$$

The nonlinear behaviour of the core material can be introduced using a core block with variable permeance. Its value is given as

$$
P(H)=\mu(H) \cdot \frac{A}{l}
$$

where the cross section area $A$ and magnetic path length $l$ are geometry-related constant parameters, while the permeability $\mu(H)$ accounts for the material characteristic which should be continuously updated during the simulation depending on the field strength $H$. The internal structure of the core block with hysteresis effect modeled by classical Preisach is shown in Fig. 2. This block is already provided by the system-level simulation software PLECS for power electronics [2]. The magnetomotive force $F$ between two terminals is divided by $l$ and provided as field strength input $H$ to the C-Script block, inside which $\mu H$ is calculated following the formulation introduced by [5]. The $\mu(H)$ output is then multiplied by $A / l$ and fed to the variable core block. Since the differential permeance value is calculated, the $d P / d t$ input of the variable core block can be set to zero. The third input magnetic flux $\Phi$ is provided as integration of the flux rate.

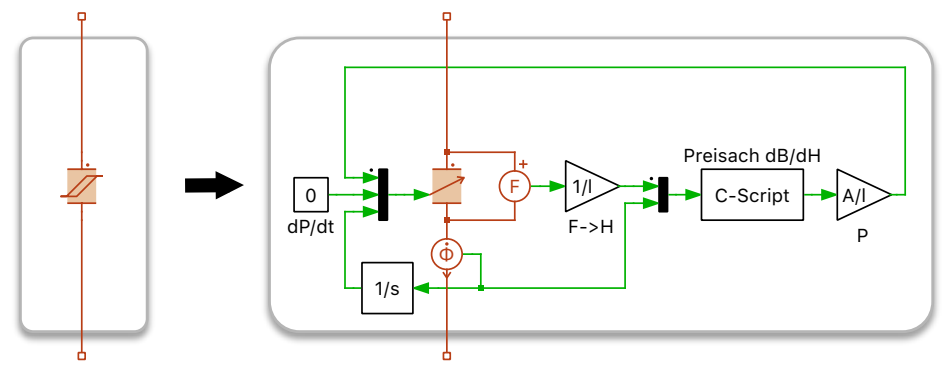

Fig. 2: Structure of hysteresis permeance block using variable core block and C-Script for classical Preisach model 


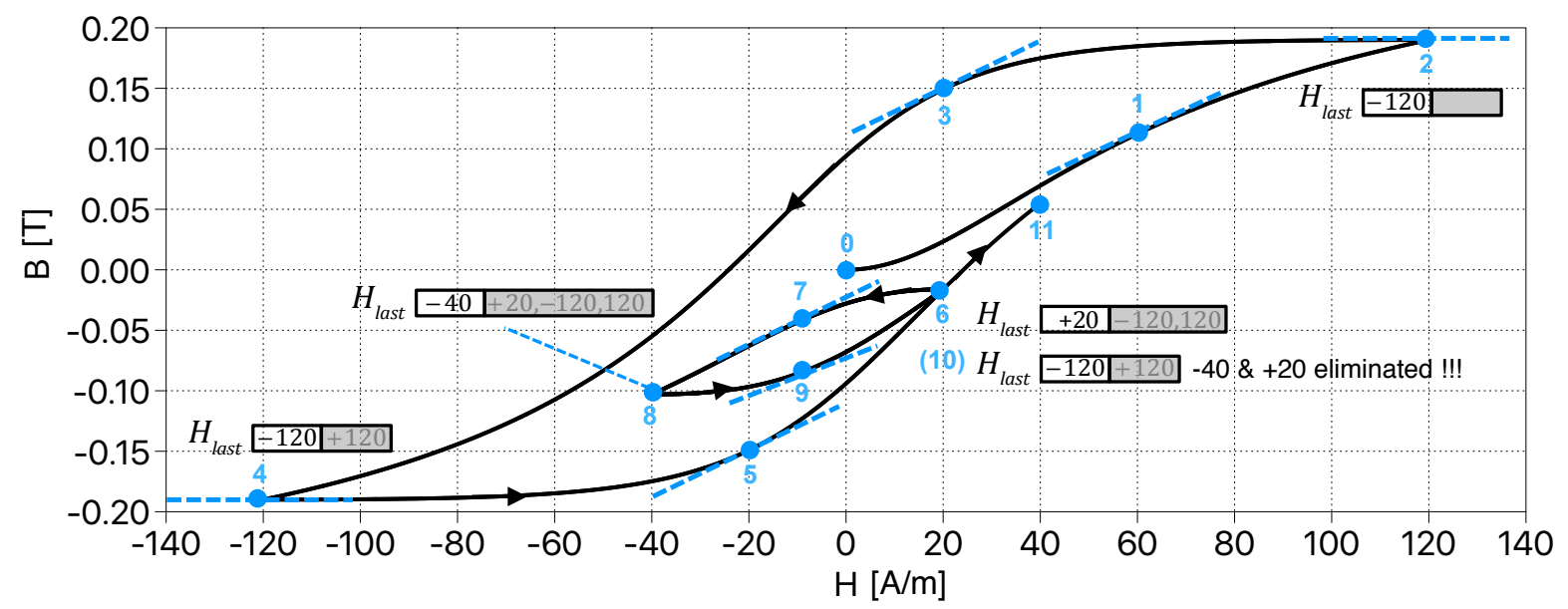

Fig. 3: Simulation of hysteresis loop starting from origin using classical Preisach model

Fig. 3 illustrates the scenario of a hysteresis loop simulation in a permeance-capacitor based magnetic circuit:

- When the simulation starts, the operation point initiates from the origin of the B-H plane and moves along the virgin curve. There the permeability, or in other words, slope of the curve $d B / d H$ at given field strength $H$ (e.g. on position 1) is calculated as

$$
\mu_{\uparrow, \operatorname{Virgin}}(H)=\frac{K}{1+\left(\frac{H-H_{0}}{\sigma}\right)^{2}} \cdot K \cdot \sigma \cdot\left[-\arctan \left(\frac{-H-H_{0}}{\sigma}\right)+\arctan \left(\frac{H-H_{0}}{\sigma}\right)\right]
$$

where $K, H_{0}$ and $\sigma$ are model parameters that need to be identified for different materials.

- At $H=+120$ (position 2) the external excitation flips polarity so that $H$ starts to decrease. The permeability on the descending branch (e.g on position 3 ) is given as

$$
\mu_{\downarrow, H \text { last }}(H)=\frac{K}{1+\left(\frac{H-H_{0}}{\sigma}\right)^{2}} \cdot K \cdot \sigma \cdot\left[\arctan \left(\frac{-H-H_{0}}{\sigma}\right)-\arctan \left(\frac{H_{\text {last }}-H_{0}}{\sigma}\right)\right]
$$

$H_{\text {last }}=+120$ is the field strength where the excitation flips its polarity. This flip-point can be detected by the sign change of flux rate $\dot{\Phi}$ flowing through the variable core block. $\dot{\Phi}$ is provided to the second input of the C-Script block in Fig. 2 so that the C-Script block is able to record $H_{\text {last }}$ value and take it for permeability calculation. Please note that thanks to the zero-crossing detection algorithm in combination with a variable step-size solver, a small simulation step size is only required at the moment of flipping, so that the overall simulation speed is not significantly reduced.

- When the operation point reaches $H=-120$ the external excitation flips polarity to positive so that $H$ begins to increase (position 4) after that. From now on $H_{\text {last }}$ is updated to -120 , the permeability (e.g. on position 5) is given as

$$
\mu_{\uparrow, H \text { last }}(H)=\frac{K}{1+\left(\frac{-H-H_{0}}{\sigma}\right)^{2}} \cdot K \cdot \sigma \cdot\left[\arctan \left(\frac{H-H_{0}}{\sigma}\right)+\arctan \left(\frac{H_{\text {last }}-H_{0}}{\sigma}\right)\right]
$$

Please note that the previous $H_{\text {last }}=+120$ is not deleted but stored in memory of the C-Script until $H$ exceed +120 for a second time in the future. This is an important feature of the Preisach model which reflects the history-dependency of the magnetisation in reality. 
- On position 6 and later on position 8 the excitation's polarity flips twice which initiate one descending- and one ascending branch successively, so that a minor loop is generated. The calculation of the permeability on the descending- (e.g. position 7) and ascending- (e.g. position 9) branch of the minor loop follows equation (4) and (5), with $H_{\text {last }}$ equal to +20 and -40 , respectively. Please note that from the position 8 on until position 10 (overlaps with position 6), there are in total values of four $H_{\text {last }}$ stored in the memory. When the operation point passes position 10, $H_{\text {last }}=+20$ and $H_{\text {last }}=-40$ are removed from the memory. The permeability after that (e.g. on position 11) is calculated using equation (5) with $H_{\text {last }}=-120$, which is the same as position 5.

\section{Combined Model}

The classical Preisach model is able to fit well wide hysteresis loops. For soft materials with narrow hysteresis loop, however, it will result in a distorted shape. In Fig. 4(a) the measured hysteresis loop of ferrite type N87 in the range $H \in[-120,+120]$ is compared to the one simulated with the classical

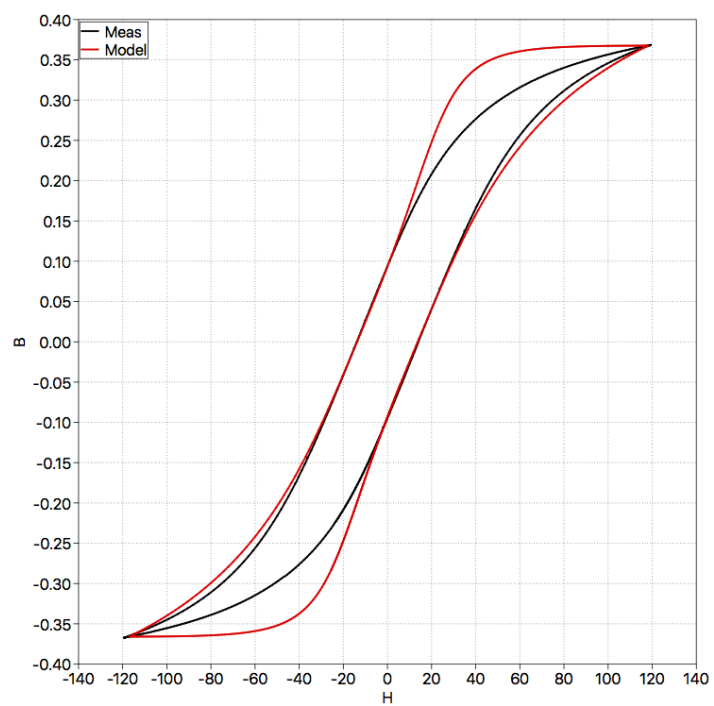

(a)

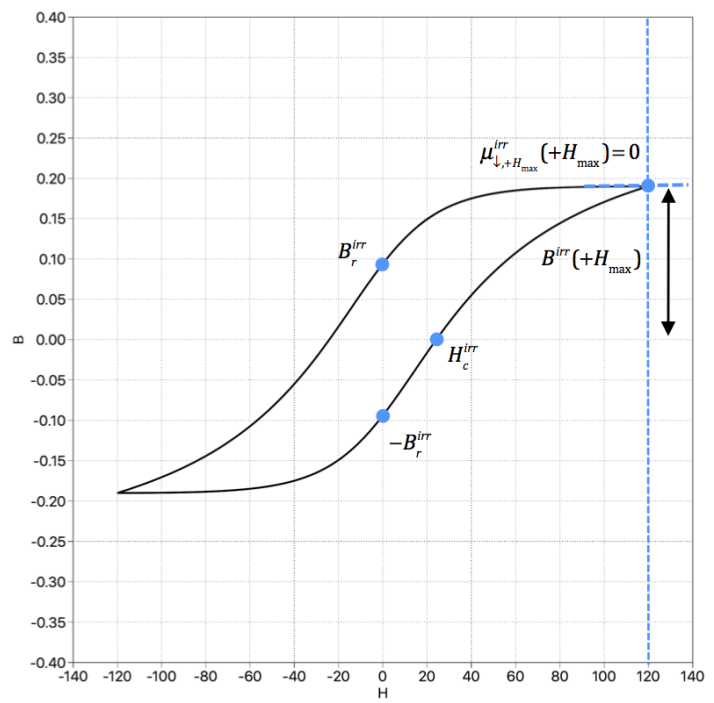

(c)

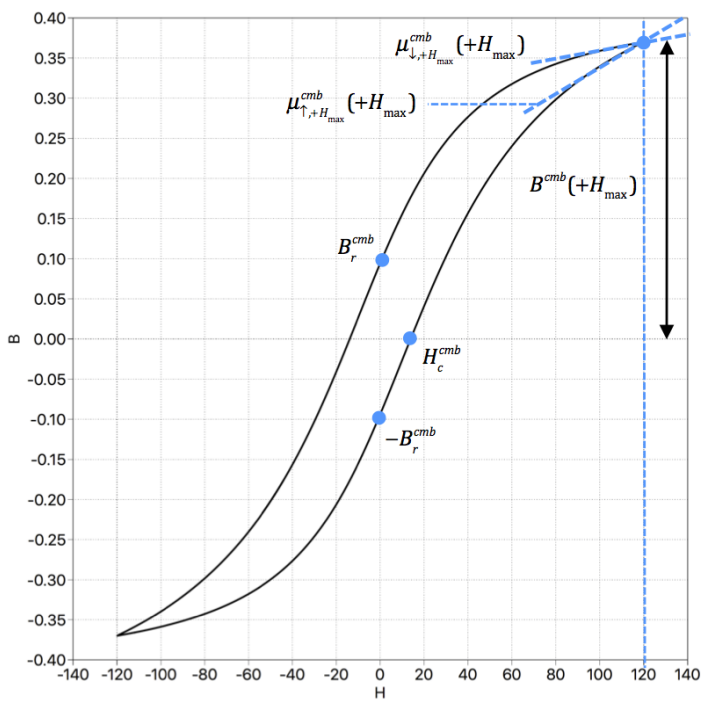

(b)

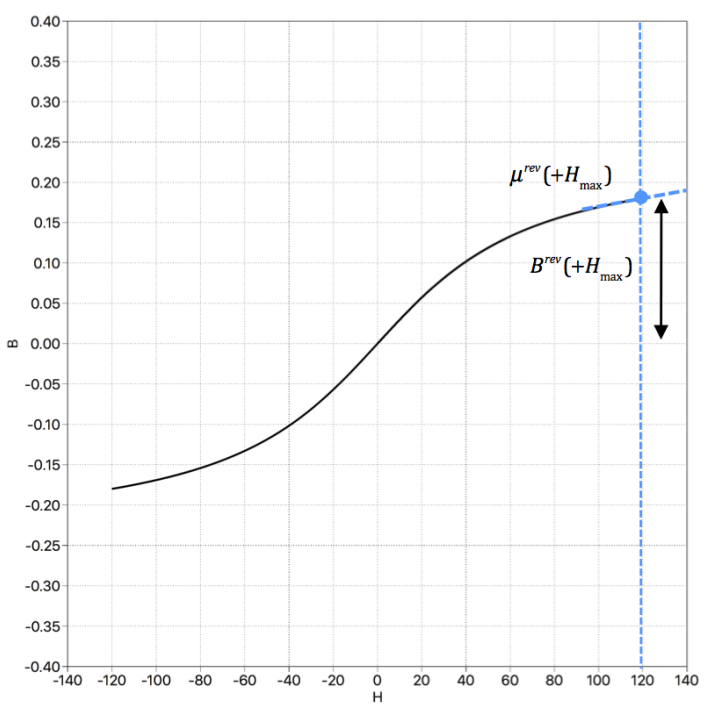

(d)

Fig. 4: Hysteresis loop of classical Preisach model and combined hysteresis loop with its irreversible and reversible component for N87 material (a) Distorted hysteresis loop of classical Preisach model (b) Hysteresis loop of combined model (c) Irreversible component of classical Preisach model (d) Reversible component 


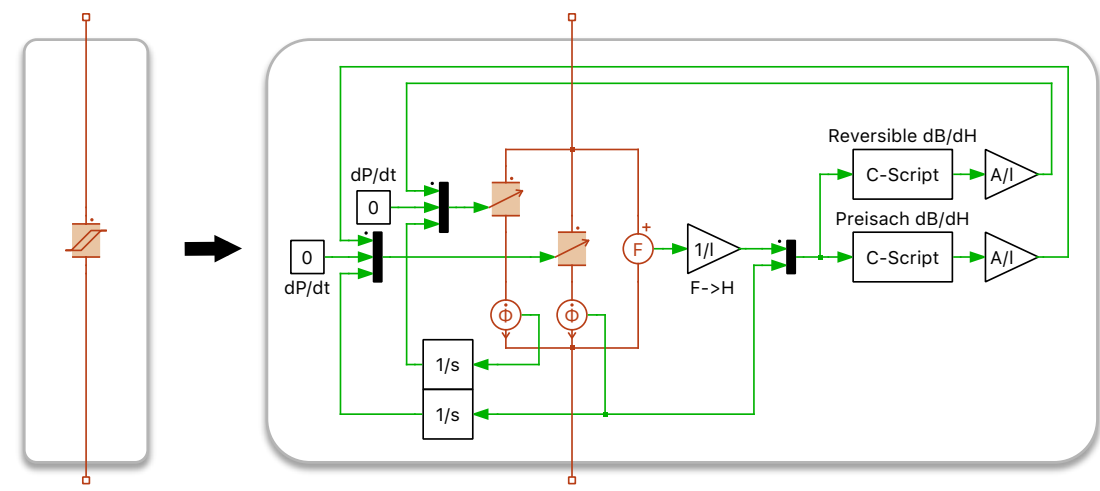

Fig. 5: Structure of hysteresis permeance block using variable core block and C-Script blocks for both irreversible classical Preisach model and reversible component

Preisach model. The parameters $K, H_{0}$ and $\sigma$ are identified via fitting the peak point $\left(H_{\max }, B_{\max }\right)$, remanent flux density $B_{r}$ and coercitive field strength $H_{c}$. Although the model is able to reproduce the same $\left(H_{\max }, B_{\max }\right), B_{r}$ and $H_{c}$, a large mismatch can be observed on the remaining part of the hysteresis loop. To solve this problem, the simulated hysteresis loop in Fig. 4(b) can be composed of two components: a irreversible hysteresis loop modeled by classical Preisach (Fig. 4(c)) and a reversible B-H curve (Fig. 4(d)), following the approach from [7]. The combined flux density $B^{c m b}$ is the summation of the two components

$$
B^{c m b}(H)=B^{i r r}(H)+B^{r e v}(H)
$$

and so is the permeability $\mu^{c m b}$

$$
\mu^{c m b}(H)=\mu^{i r r}(H)+\mu^{r e v}(H)
$$

The structure of the proposed combined hysteresis model in a permeance-capacitance based magnetic circuit is shown in Fig. 5. Two variable core blocks are connected in parallel, to account for the classical Preisach model and reversible component. The permeance of the two core blocks are calculated in two $\mathrm{C}$-Script blocks and scaled with $A / l$. The equivalent permeance of the whole hysteresis core block is essentially the sum of the individual permeances:

$$
P^{c m b}(H)=\mu^{i r r}(H) \cdot \frac{A}{l}+\mu^{r e v}(H) \cdot \frac{A}{l}
$$

The output of the C-Script block "Preisach dB/dH" ( $\mu^{\text {irr }}$ ) is obtained from equations (3) - (5). For the reversible part, the inverse trigonometric (arctan) function proposed by [10] and hyperbolic cotangen (coth) function by [11], [12] can be adopted. The derivatives of these functions $\left(\mu^{r e v}(H)\right)$ are calculated by the other C-Script block as

$$
\begin{aligned}
& \mu_{\mathrm{arctan}}^{r e v}(H)=\frac{d B^{r e v}}{d H}(H)=B_{S} \cdot \frac{2}{\pi} \cdot \frac{1}{\alpha \cdot\left(1+(H / \alpha)^{2}\right)} \\
& \mu_{\mathrm{coth}}^{r e v}(H)=\frac{d B^{r e v}}{d H}(H)=B_{S} \cdot\left(\frac{\tanh ^{2}(H / \alpha)-1}{\alpha \cdot \tanh ^{2}(H / \alpha)}-\frac{\alpha}{H^{2}}\right)
\end{aligned}
$$

where $B_{s}$ and $\alpha$ are parameters. The parameter identification of the combined hysteresis model can be carried out using an experimentally measured hysteresis loop, which is symmetrically located around zero and spreads from $-H_{\max }$ to $+H_{\max }$. With the parameter $\alpha$ and the permeability at beginning of descending branch $\left(\mu_{\downarrow+H_{\text {max }}}^{c m b}\left(+H_{\text {max }}\right)\right.$ in Fig. 4(b)) from a measured hysteresis loop, the parameter $B_{s}$ can be directly calculated as 


$$
B_{S}=\mu_{\downarrow+H_{\text {max }}}^{c m b}\left(+H_{\max }\right) \cdot \frac{\pi}{2} \alpha\left(1+(H / \alpha)^{2}\right)
$$

Please note that the expression above exploits the fact that the permeability of the irreversible component modeled by classical Preisach at the beginning of a descending branch $\left(\mu_{\downarrow,+H_{\max }}^{i r r}\left(+H_{\max }\right)\right.$ in Fig. 4(c) $)$ is always equal to zero. With $\alpha$ and $B_{s}$ of the reversible component obtained, three characterisation points of the irreversible hysteresis loop spreading the range $H \in\left[-H_{\max },+H_{\text {max }}\right]$ can be extracted, as marked in Fig. 4(c):

- The maximum flux density $B^{i r r}\left(+H_{\max }\right)$ can be calculated as

$$
B^{i r r}\left(+H_{\max }\right)=B^{c m b}\left(+H_{\max }\right)-B^{r e v}\left(+H_{\max }\right)
$$

- The remanence flux density $B_{r}^{i r r}$ at $H=0$ is equal to that on the measured hysteresis loop

$$
B_{r}^{i r r}=B_{r}^{c m b}
$$

- The coercive permeability $H_{c}^{i r r}$ scan be decided from the equation below, under the assumption that the B-H characteristic between the remanence- and coercive- point is approximately linear.

$$
B^{r e v}\left(H_{c}^{i r r}\right)=\frac{H_{c}^{i r r}-H_{c}^{c m b}}{H_{c}^{c m b}} \cdot B_{r}^{c m b}
$$

Based on $B^{i r r}\left(+H_{\max }\right), B_{r}^{i r r}$ and $H_{c}^{i r r}$ obtained above, the three parameters $K, H_{0}$ and $\sigma$ of the classical Preisach model can be identified using a fitting algorithm, e.g. f minsearch function provided by Matlab. Please note that the parameter $\alpha$ of the reversible component can be adjusted to fit the permeability at the end of the ascending branch $\left(\mu_{\uparrow,+H_{\max }}^{c m b}\left(+H_{\max }\right)\right.$ in Fig. 4(b)). The workflow of complete parameter identification can be organised as a two-loop structure as illustrated in Fig. 6(a), aiming to minimise two objective functions which are defined as the quadratic error between model and measurements:

$$
\begin{aligned}
& f_{1}\left(K, H_{0}, \sigma\right)=\left(\frac{B_{\text {model }}^{\text {irr }}\left(+H_{\text {max }}\right)-B_{\text {meas }}^{\text {irr }}\left(+H_{\text {max }}\right)}{B_{\text {meas }}^{\text {irr }}\left(+H_{\text {max }}\right)}\right)^{2} \\
& +\left(\frac{B_{r, \text { model }}^{\text {irr }}-B_{r, \text { meas }}^{\text {irr }}}{B_{r, \text { meas }}^{\text {irr }}}\right)^{2}+\left(\frac{H_{c, \text { model }}^{\text {irr }}-H_{c, \text { meas }}^{\text {irr }}}{H_{c, \text { meas }}^{\text {irr }}}\right)^{2} \\
& f_{2}(\alpha)=\left(\frac{\mu_{\uparrow,+H_{\text {max }}, \text { model }}^{\text {cmb }}\left(+H_{\text {max }}\right)-\mu_{\uparrow,+H_{\text {max }}, \text { meas }}^{\text {cmb }}\left(+H_{\text {max }}\right)}{\mu_{\uparrow,+H_{\text {max }}, \text { meas }}^{c m b}\left(+H_{\text {max }}\right)}\right)^{2}
\end{aligned}
$$

\section{Validation}

For the validation of the proposed modelling approach, a test bench based on the structure described in [13] has been established to measure the hysteresis loop of the core materials, as shown in Fig. 6(b). The test setup is composed of a RF power amplifier (gain-bandwidth product $2 \mathrm{MHz}$ ) to generate arbitrary voltage waveform, as well as a control unit (PLECS RT-Box) to provide teh reference signal and measurement data access. As this work focus on the frequency independent hysteresis effect without eddy current, the power amplifier's output voltage has been configured as low frequency $50 \mathrm{~Hz}$ sinusoidal wave. As the temperature dependency is not the scope of this work, all tests have been carried out under room temperature of $25^{\circ} \mathrm{C}$.

The PLECS simulation model (Fig. 7(a)) is composed of the hysteresis core block which represents the core sample, the power amplifier as an ideal sinusoidal AC voltage source and the equivalent resistance (including the shunt resistor for current measurement and parasitics). All components are configured to match the test bench hardware. The ferrite material N87 from TDK is taken as the first verification 


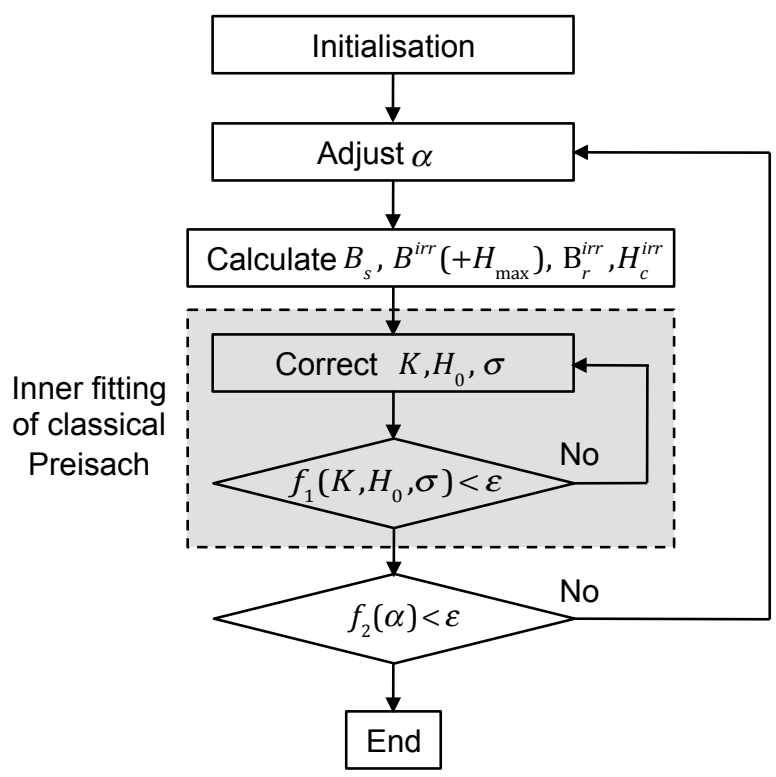

(a)
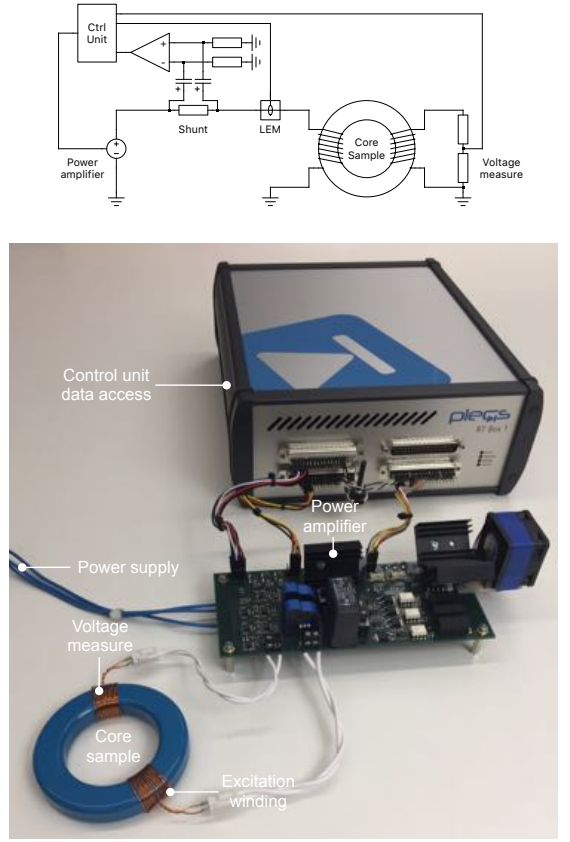

(b)

Fig. 6: (a) Workflow of parameter identification (b) Hardware configuration of the test setup

case, where the toroidal core of size code "R 87.0x54.3x13.5" is taken as sample. The material-related parameters are identified by fitting the measured hysteresis loop with $H_{\max }=120 \mathrm{~A} / \mathrm{m}$. Please note that these parameters will remain the same in the other verification cases. In Fig. 7(a) and Fig. 7(b), the time domain waveforms of the primary winding current and the secondary winding voltage as well as the hysteresis loop at $H_{\text {peak }}=100 \mathrm{~A} / \mathrm{m}$ from measurement and simulation using the arctan function are compared with each other. Although an ideal sinusoidal voltage is generated on the power amplifier output, the measured primary winding current and the secondary open-circuit voltage, are heavily distorted, due to the presence of nonlinear hysteresis effect. The proposed simulation model is able to approximate
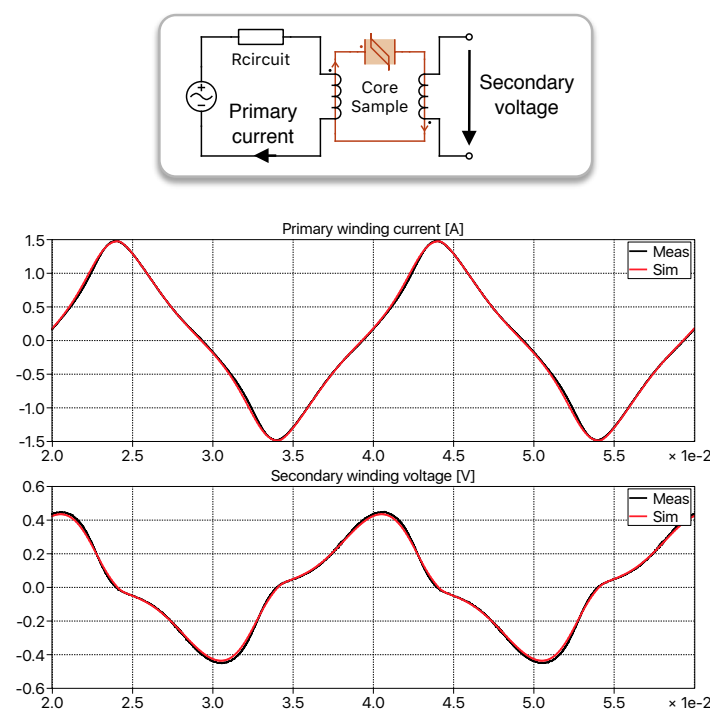

(a)

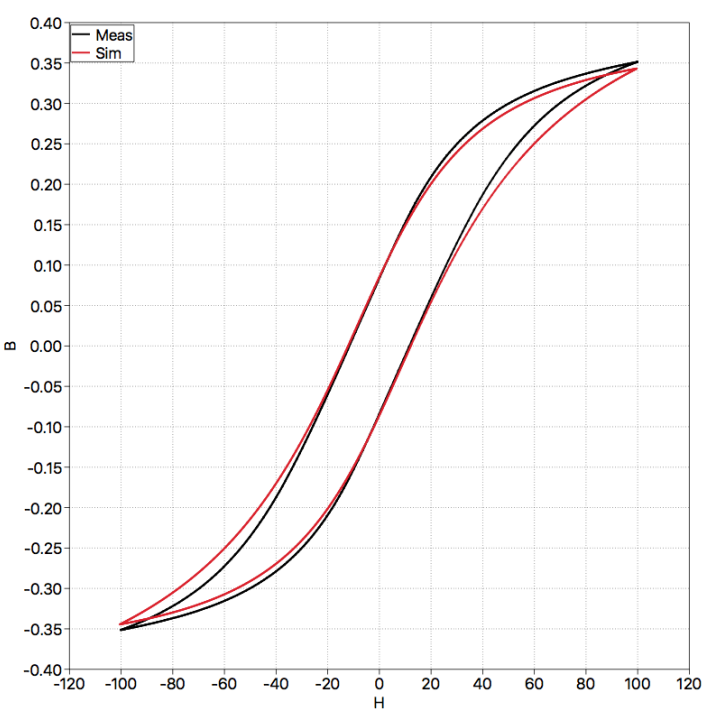

(b)

Fig. 7: Comparison between measurement and simulation of Ferrite N87 (a) Simulation model and time-domain primary winding current and secondary voltage waveform at $H_{\text {peak }}=100 \mathrm{~A} / \mathrm{m}$ (b) Hysteresis loop at $H_{\text {peak }}=$ $100 \mathrm{~A} / \mathrm{m}$ 


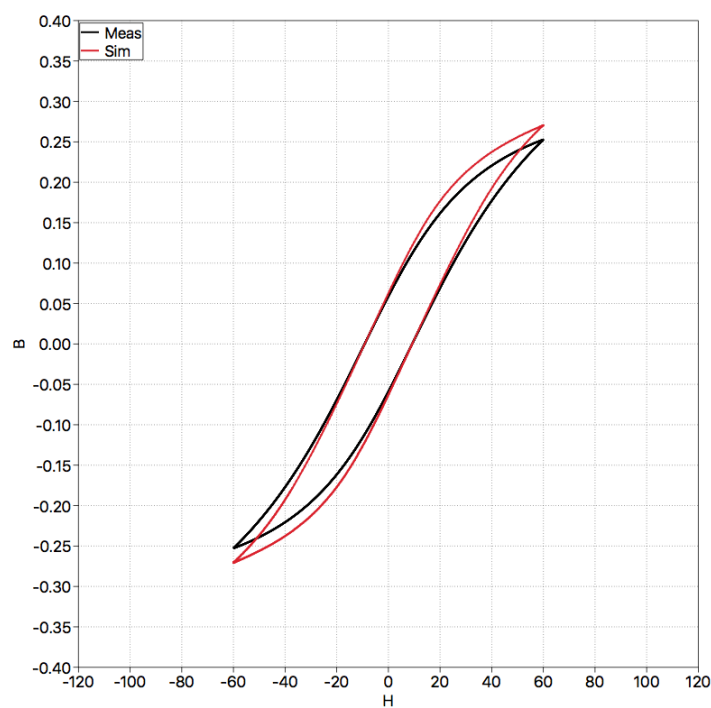

(a)

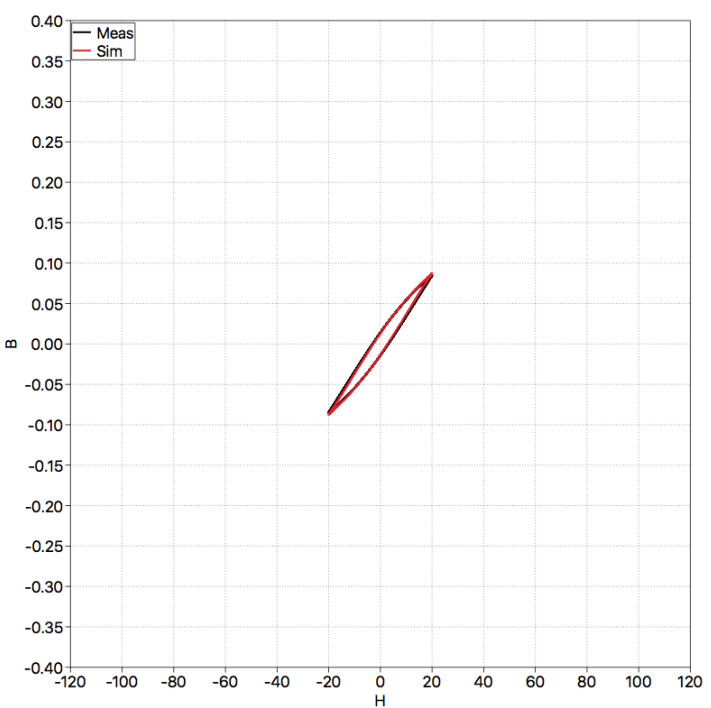

(b)

Fig. 8: Comparison between measurement and simulation of Ferrite N87 (a) Hysteresis loop at $H_{p e a k}=60 \mathrm{~A} / \mathrm{m}$ (b) Hysteresis loop at $H_{\text {peak }}=20 \mathrm{~A} / \mathrm{m}$

the time domain waveform as well as the hysteresis loop well. Multiplying the primary winding current and the secondary winding voltage, then averaging the result in a time window of $0.02 \mathrm{~s}$, yields the core loss of the sample (without resistive winding). The measured value is $0.038 \mathrm{~W}$ and simulation result is $0.040 \mathrm{~W}$, which indicates less than $10 \%$ error. The hysteresis loop at other values of $H_{\text {peak }}$ are compared and yield a good match between simulation and measurement according to Fig. 8(a) and 8(b).

In the second validation case, the nanocrystaline material VITROPERM 250F from VAC is evaluated, where the toroidal core of size code "W 982-02" is take as a sample. The comparison between measurement and simulation are shown in Fig. 9. The hysteresis loop of the nanocrystaline material is significantly thinner than that of the ferrite material, which indicates that the reversible component of this type of material dominates more in the B-H characteristic. Here again, the simulation model is able to reproduce the material nonlinearity.

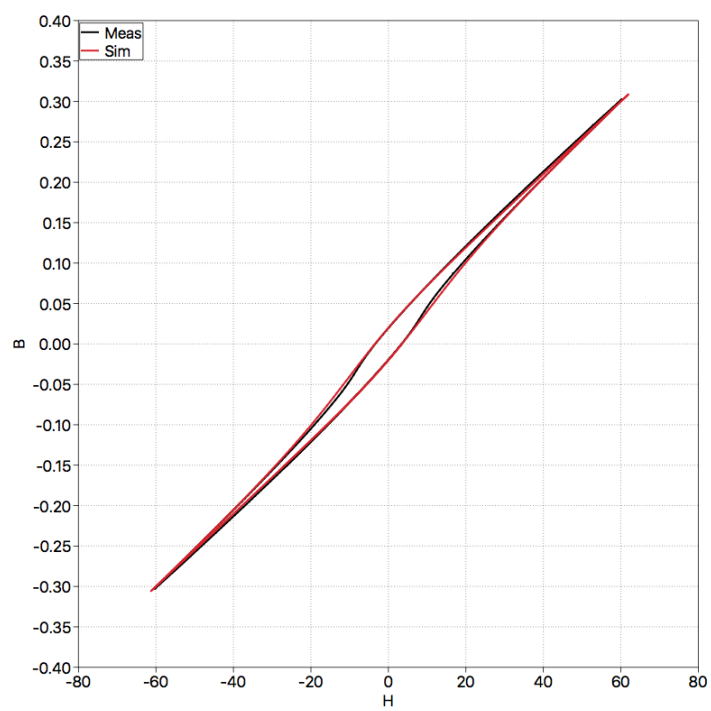

(a)

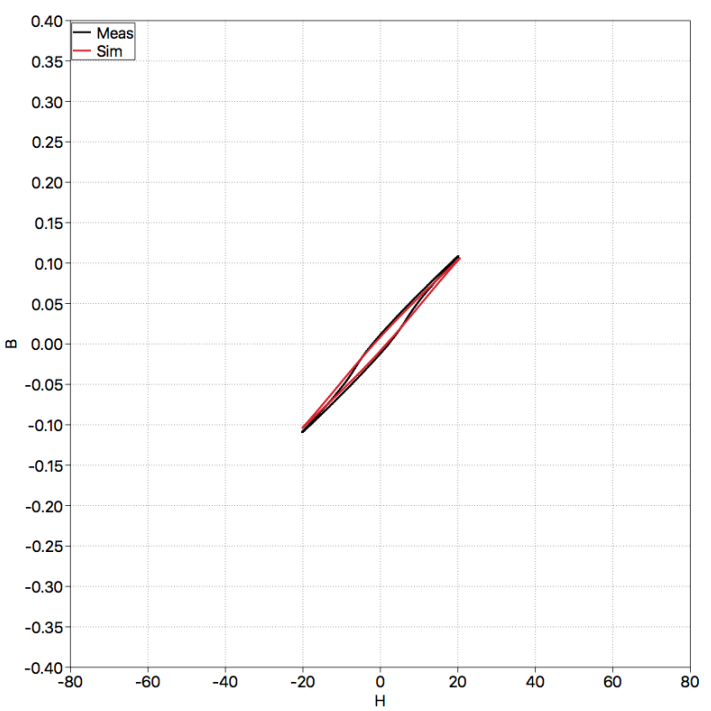

(b)

Fig. 9: Comparison between measurement and simulation of nanocrystaline VITROPERM 250F (a) Hysteresis loop at $H_{\text {max }}=60 \mathrm{~A} / \mathrm{m}$ (b) Hysteresis loop at $H_{\max }=20 \mathrm{~A} / \mathrm{m}$ 


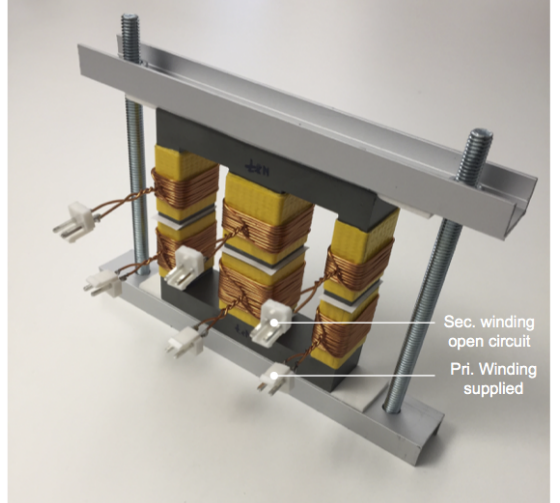

(a)

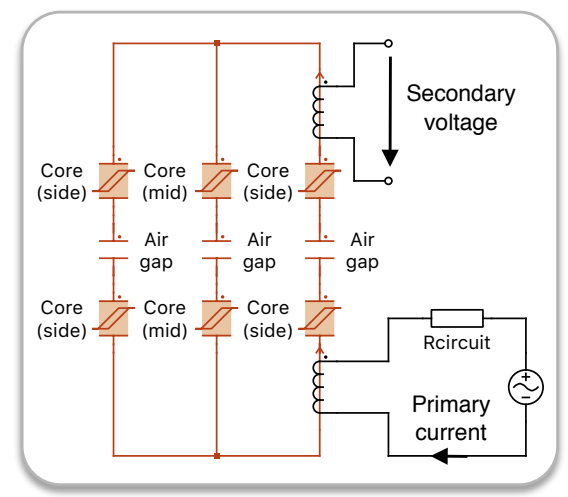

(b)

Fig. 10: Verification case of an E-core transformer with one winding on the side limb supplied and voltage of another on the same limb measured (a) Photo of the E-core transformer (b) Simulation model of the E-core transformer

After the material-related parameters of the core block are identified, one can configure geometrical parameters as well as connect multiple core blocks together, in order to model cores of arbitrary geometry. For verification, a prototype transformer has been constructed using E-core from EPCOS of size "E80/38/20" and material N87, as shown in Fig. 10(a). An air gap of 0.075mm has been introduced in each of the limbs. Two windings are installed on the side-limb, the lower one with $N_{1}=15$ turns is supplied by the power amplifier output of the test bench, while the upper one with $N_{2}=10$ turns is left open and its voltage is measured. The magnetic circuit model of the prototype transformer is established as demonstrated in Fig. 10(b). Hysteresis core blocks using the proposed model for N87 have been adopted to construct the magnetic flux path inside the E-core. The geometry-related parameters $(A$ and $l$ ) are configured according to the size of the mid- and side- limbs, respectively. The three air gaps are represented by linear permeance blocks. In Fig. 11(a) the simulated primary winding current and the secondary winding voltage are compared between experimental measurement and simulation: The simulation model is able to reproduce the measurement with a minor error. Compared to Fig. 7(a), the waveforms in this verification case have significantly lower harmonic content. This is due to the presence of the air gap, which reduces the contribution from the nonlinear hysteresis effect of the transformer core. The core loss here can be calculated via multiplying the primary winding current and the secondary

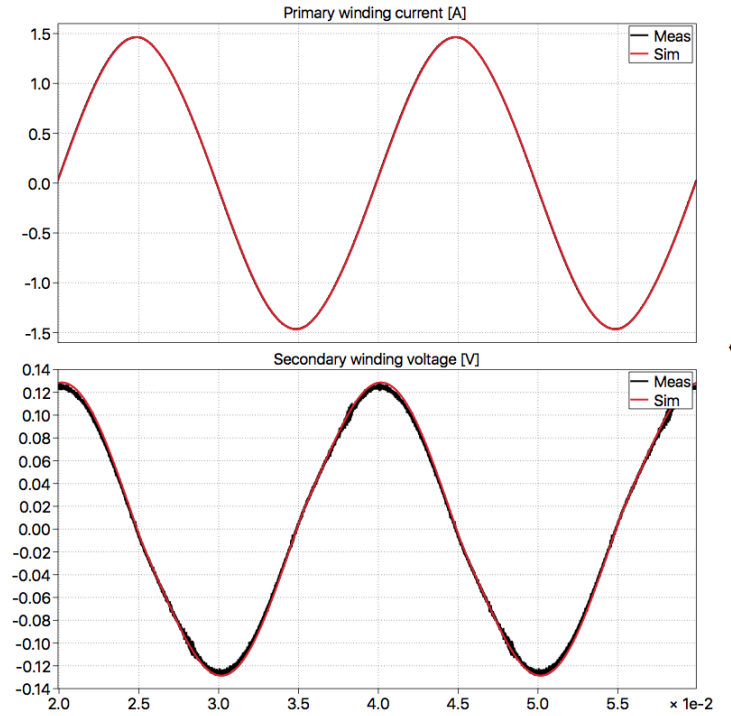

(a)

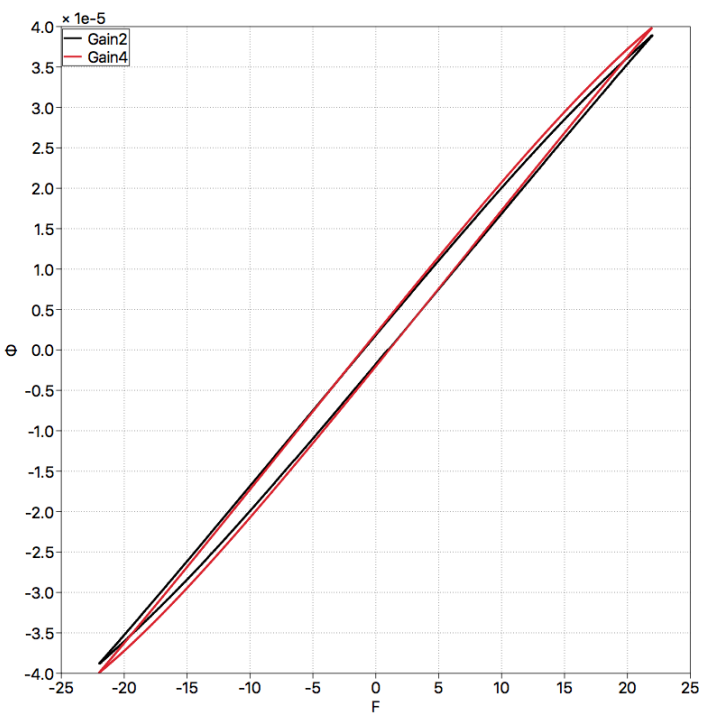

(b)

Fig. 11: Comparison between measurement and simulation of E-core transformer (a) Primary winding current and secondary winding voltage (b) $\Phi-F$ plot 
winding voltage divided by the turns ratio $\left(N_{1} / N_{2}\right)$. This yields $0.0025 \mathrm{~W}$ for measurement and $0.0028 \mathrm{~W}$ for simulation, corresponding to an error of $12 \%$. In Fig. 11(b), the magnetic flux $\Phi$ (in Henry) of the side limb and magneto motive force $F$ (in $A$ ) generated by the primary winding $\left(F=I \cdot N_{1}\right)$ are plotted together reflecting the combined behaviour of the linear air gap and nonlinear core material. Again, the proposed model is able to match the measurement well.

\section{Conclusion}

This work has proposed a permeance-capacitance based magnetic circuit model for the hysteresis effect of soft core materials. In comparison to existing publications, a simplified identification process of the material related parameters has been introduced as well, based on the experimentally measured hysteresis loop. In combination with an electrical circuit, the proposed model is able to reproduce the symmetrical hysteresis loops of ferrite and nanocrystaline materials accurately as well as the distorted current and voltage waveform which arise from this nonlinearity in time domain simulation. When the geometryrelated parameters of the core blocks are determined, one can establish a magnetic circuit model of arbitrary core geometries and simulate the core loss as well as the related harmonic distortions with good accuracy. Future work will include modeling of unsymmetrical minor hysteresis loops that occurs due to DC-bias on both field strength and flux density, as this is the case in grid-connected inverters and DC-DC converters without transformer isolation.

\section{References}

[1] D. Hamill. "Lumped equivalent circuits of magnetic components: the gyrator-capacitor approach". In: IEEE Transactions on Power Electronics. Vol. 8. 1994, pp. 97-103.

[2] J. Allmeling, W. Hammer, and J. Schönberger. "Transient simulation of magnetic circuits using the permeance-capacitance analogy”. In: Control and Modeling for Power Electronics (COMPEL), IEEE 13th Workshop on. 2012.

[3] M. Luo and D. Dujic. "Permeance based modelling of the core corners considering magnetic material nonlinearity”. In: Annual Conference of the IEEE Industrial Electronics Society (IECON). 2015, pp. 950955.

[4] M. Luo, D. Dujic, and J. Allmeling. "Leakage flux modelling of multi-winding transformer using permeance magnetic circuit". In: 2016 IEEE Applied Power Electronics Conference and Exposition (APEC). 2016, pp. 1108-1114.

[5] B. Azzerboni et al. "Remarks about Preisach function approximation using Lorentzian function and its identification for non-oriented steels". In: IEEE Transactions on Magnetics. Vol. 39. 5. 2003, pp. 30283030 .

[6] J. Liu and V. Dinavahi. "Detailed magnetic equivalent circuit based real-time nonlinear power transformer model on FPGA for electromagnetic transient studies". In: IEEE Transactions on Industrial Electronics. Vol. 63. 2. 2016, pp. 1191-1202.

[7] E. D. Torre. Magnetic Hysteresis. IEEE Press, 1999.

[8] E. Cardelli, E. D. Torre, and E. Pinzaglia. "Identifying the Preisach function for soft magnetic materials". In: IEEE Transactions on Magnetics. Vol. 39. 3. 2003, pp. 1341-1344.

[9] M. Pasquale and G. Bertotti. "Application of dynamic Preisach model to the simulation of circuits coupled by soft magnetic cores”. In: IEEE Transactions on Magnetics. Vol. 32. 5. 1996, pp. 4231-4233.

[10] C. Perez-Rojas. "Fitting saturation and hysteresis via arctangent funcitons". In: IEEE Power Engineering Review. Vol. 20. 1. 2000, pp. 55-57.

[11] D. C. Jiles and D. L. Atherton. "Ferromagnetic hysteresis". In: IEEE Transactions on Magnetics. Vol. 19. 6. 1983, pp. 2183-2185.

[12] D. C. Jiles, J. B. Thoelke, and M. K. Devine. "Numerial determination of hystereiss parameters for the modeling of magnetic properties using the theory of ferrormangnetic hysteresis". In: IEEE Transactions on Magnetics. Vol. 28. 1. 1992, pp. 27-35.

[13] D. Tan, J. L. Vollin, and S. M. Cuk. “A practical approach for magnetic core-loss characterization”. In: IEEE Transactions on Power Electronics. Vol. 10. 2. 1995, pp. 124-130. 